\section{Glanzrhodiumbad erzeugt brillant weiße, dekorative Schichten}

M it dem Glanzrhodiumbad JE88 Go vergrößert die Firma C. Jentner ihr Spektrum an galvanischen Bädern um ein Produkt, das brillant weiße, glänzende und abriebfeste dekorative Schichten mit einer Dicke von bis zu 1,5 $\mu \mathrm{m}$ erzeugt. Die Oberflächen erreichen $L^{*}$-Werte zwischen 90,5 und 91 und eignen sich damit für Schmuck, Brillengestelle und Uhren. Mit dem Rhodiumelektrolyt lassen sich auch Silberprodukte effizient gegen das Anlaufen schützen. Das Bad ist gebrauchsfertig im 1-Liter-Behälter sowie als Konzentrat erhältlich. Als besonderen Service bietet der Herstel- ler die Aufbereitung gebrauchter Lösungen oder der KI a rspüle an. So lassen sich wertvolle Edelmetalle zurückgewinnen. Dieser Service steht für Flüs-

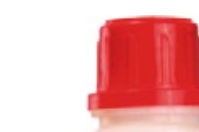
sigkeitsmengen ab 10 I zur Verfügung. Tel. 07231 28098-0, www.jentner.de

\title{
Wartungsarme Filteranlage für Plasmastäube
}

Der hat mit der PlasmaFil eine Filteranlage für die Beseitigung großer Staubmengen entwickelt, die beim Plasmaschneiden frei werden. Das Gerät zeichnet sich durch eine lange Lebensdauer und Filterstandzeit aus.

Nach Angaben des Herstellers müssen keine Filterwechsel durchgeführt werden. Herzstück des neuen Systems ist ein Plattenfilter mit einer speziellen Beschichtung, der selbst ultrafeine Staubpartikel einfängt. Anders als üblich ist der Filter als Platte und nicht als Zylinder konstruiert. Dadurch wird verhindert, dass die einzelnen Falten des Filters verkleben. Damit steht die gesamte Filterfläche für den Filterprozess zur Verfügung. Die Filteranlage verfügt über einen SparkTrap Funkenvorabscheider. Dieser fängt Funken ab und verhindert damit Filterbrände. Zum anderen filtert der Vorabscheider grobe Stäube heraus, was den Filter entlastet. Das Gerät kommt ohne die üblichen Staubsammelbehälter aus. Stattdessen wurde es mit einer vakuumbasierten Entsorgung ausgestattet. Der Staub wird in einem großen BigBag gesammelt. Dieser ermöglicht eine kontaminationsfreie Entsorgung und kann anders als gewöhnliche Behälter während des Schneidprozesses gewechselt werden, ohne dass die Arbeit unterbrochen werden muss. Dank des gedämpften Ventilators ist die Lärmbelastung gering.

Tel. 02564 68-0, www.kemper.eu
Sicherheits-Filterdeckel schützt bei der Wartung automatischer Reinigungsanlagen

Die e Wartung automatisch betriebener Reinigungsanlagen hat ihre Tücken. Läuft die Anlage während der Wartung an, können sich die Arbeiter verletzten. In anderen Fällen kann heißes Reinigungsmedium aus dem Filtergehäuse schießen und zu Verbrühungen oder Verätzungen führen. Um das zu verhindern hat die Firma BvL einen Sicherheits-Filterdeckel für vollautomatisierte Reinigungsanlagen entwickelt. Der Filterdeckel eignet sich besonders für Anlagen, die über eine automatisch zeitgesteuerte Tankspülung verfügen, da die Pumpe während eines Filterwechsels anlaufen könnte. Sinnvoll ist der Einsatz auch bei Anlagen mit Umschaltfiltern, die automatisch auf den Standby-Filter umschalten. Hier ist es denkbar, dass die Anlage auf ein für den Filterwechsel geöffnetes Filtergehäuse umschaltet. Die Deckel bieten sich darüber hinaus für Anlagen an, bei denen das Filtergehäuse versehentlich geöffnet werden könnte, während die Pumpe läuft. Vermieden werden auch Verletzungen, die beim automatischen Starten einer Reinigungsanlage aus dem Standby-Betrieb auftreten können, beispielsweise bei Inline-Durchlaufanlagen. Die Sicherheits-Filterdeckel eignen sich für Neuanlagen und zum Nachrüsten von älteren Anlagen des Herstellers. Tel. 05903 951-60, www.bvl-group.de

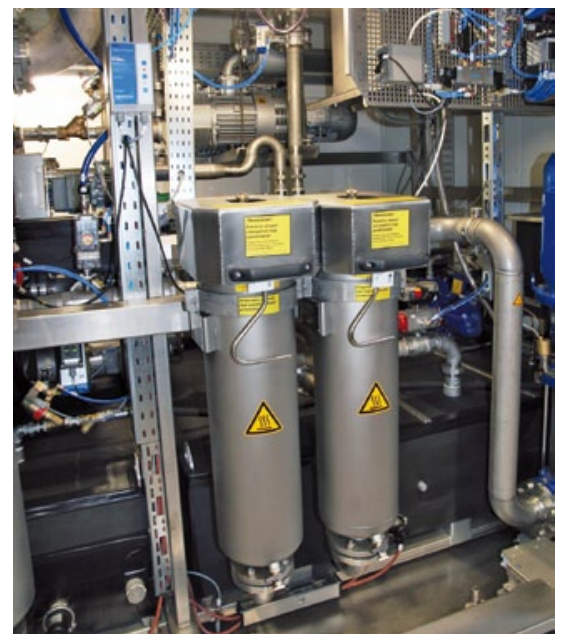

